\title{
Liaison et conscience phonologique en Côte d'Ivoire: analyse des réponses épi- et métaphonologiques face à un test d'acceptabilité administré aux adultes abidjanais
}

\author{
Giulia De Faviis \\ Università di Roma La Sapienza, Circonvallazione Tiburtina 4, Italia
}

\begin{abstract}
Résumé. Par le biais d'un test d'acceptabilité phonologique on se propose d'étudier la perception subjective explicite qu'une population de 22 étudiants universitaires ivoiriens déclare avoir au sujet de la liaison, un phénomène complexe qui dépend de la combinaison de différents facteurs linguistiques et extralinguistiques. Dans un premier temps, on a demandé aux informateurs de détecter des incongruités lors de l'écoute d'un texte lu qui présentait des liaisons déviantes: cette tâche visait la sollicitation des réactions épiphonologiques; dans un deuxième temps, on leur a demandé de juger de l'acceptabilité des incongruités repérées et d'argumenter leurs réponses: cette tâche visait la sollicitation des réactions métaphonologiques. L'observation du corpus abidjanais et sa comparaison avec une enquête parisienne analogue (Celata, De Flaviis et Floquet, à paraître), vont nous permettre: de vérifier si les réactions épiphonologiques des locuteurs ivoiriens correspondent à la tripartition en liaisons catégoriques, variables et interdites telle que la montre le corpus PFC et d'observer s'il y a un effet de population; de décrire la diversité des motivations sous-jacentes aux pratiques langagières du phénomène en Côte d'Ivoire; de considérer les appréciations naïves comme un outil informatif à intégrer aux connaissances actuelles sur la liaison découlant de la phonologie de corpus.
\end{abstract}

\footnotetext{
Abstract. French liaison and phonological awareness in Côte d'Ivoire : an analysis of epiphonological and metaphonological answers to an acceptability judgment test administrated on native university students in Abidjan. Via an acceptability judgment test administrated on 22 university students in Abidjan we will study the explicit subjective perception that native Ivorian speakers have about French liaison, a complex phenomenon of French phonology whose
} 
realization depends on the combination of linguistic and extralinguistic factors. Firstly, we asked the participants to listen to an oral passage including a number of deviant realizations of liaison and to spot the pronunciations that they considered anomalous: the aim of this task was to observe the informants' epiphonological reactions. Secondly, we asked them to give an oral explanation of their answers: the aim of this task was to elicit and quantify metaphonological awareness. The analysis of the Ivorian corpus and its comparison with an analogue survey conducted in Paris (Celata, De Flaviis\&Floquet, to be published) will allow us: to investigate whether native Ivorian speakers' epilinguistic awareness of liaison correspond to the classification of liaison contexts as obligatory, optional or forbidden liaisons as showed in the PFC corpus; to investigate the presence of a population effect (Abidjan vs Paris); to describe the variety of conscious explanations and arguments underlying the use of liaison in Côte d'Ivoire; to consider the naive intuitions as an informative instrument for the current corpus data about French liaison.

\section{Introduction}

\subsection{Questions de recherche}

Le premier objectif que se pose cette étude est d'observer et quantifier, par le biais d'un test d'acceptabilité oral, la conscience phonologique que les étudiants universitaires natifs d'Abidjan ont de la liaison, un phénomène de la phonologie française dont les recherches psycholinguistiques et linguistiques ont défendu le caractère non-unitaire et complexe (Soum-Favaro et al. 2014; Eychenne \& Laks 2017; Laks et al. 2014, 2018). Sous l'expression de conscience phonologique nous entendons la capacité à faire abstraction des unités phonologiques. Cette capacité se manifesterait lors d'un traitement cognitif exigeant la manipulation ou la réponse à certains stimuli de type phonologique (le plus souvent les syllabes, les attaques, les rimes, les phonèmes) suivant différentes tâches (la détection, la substitution, l'élision, la fusion). Plusieurs travaux psycholinguistiques réalisés dans ce domaine font la distinction entre deux niveaux de traitement cognitif impliqués dans le processus d'abstraction des des unités phonologiques: l'un, épiphonologique, «qui fait référence à un traitement implicite, sans contrôle intentionnel», l'autre, métaphonologique qui «renvoie à une prise de conscience et un traitement explicite» (Ecalle, Magnan et Bouchafa 2002). Selon l'hypothèse de Ecalle, Magnan et Bouchafa 2002 :

le traitement épiphonologique ne serait que la traduction comportementale de l'état du système de connaissances phonologiques alors que le traitement métaphonologique est réalisé sous le contrôle d'un opérateur qui déclenche une procédure dont l'objet est d'extraire des unités linguistiques de la base de connaissances phonologiques pour les manipuler intentionnellement à partir d'une instruction.

En continuité avec deux travaux qu'ont réalisés Floquet et Laks (2017) et Celata, De Flaviis et Floquet (à paraître), nous allons opérer la distinction entre une «compétence épiphonologique », qui se manifeste lorsque le sujet identifie, manipule et juge les unités phonologiques de manière intuitive et irréfléchie, et une "conscience métaphonologique ", l'activité déclarative qui consiste en un retour sur ses propres intuitions superficielles et vise à les expliquer par le biais d'un commentaire explicite (Gombert 1990; La Mantia 2017). Notre expérience visera la sollicitation de ces deux traitements en fonction de deux taches différenciées. Dans un premier temps, il s'agira de susciter chez l'informateur une réponse épiphonologique : pendant qu'il écoute l'enregistrement sonore d'un texte lu par un 
locuteur natif francophone, l'enquêté est censé détecter et juger de l'acceptabilité de certaines réalisations de liaisons qui, selon notre hypothèse, pourraient être perçues comme déviantes ou peu probables si l'on faisait référence à la phénoménologie tripartite du phénomène que décrit le corpus PFC (Durand, Laks \& Lyche 2002). Dans un deuxième temps, il s'agira d'éliciter une réponse métaphonologique en demandant au sujet d'argumenter et commenter explicitement ses appréciations spontanées. Chacune de ces réponses sera ensuite quantifiée suivant la grille numérique de Pinto et El Euch (2015). Cet aspect quantitatif permet de distinguer ce protocole des autres techniques existantes conçues pour mesurer la conscience métaphonologique : à notre connaissance, les nombreux travaux visant la quantification de la conscience métaphonologique ont pour objet le plus souvent les syllabes, les rimes, les phonèmes (voir, par exemple, Chard et Dickson 1999) mais aucun d'entre eux n'aborde une catégorie spécifique telle que la liaison.

Le deuxième objectif est de comparer les données perceptives de cette étude à la distribution de la liaison en Côte d'Ivoire, notre hypothèse allant dans le sens d'un divergence entre les comportements linguistiques externes des locuteurs, observables dans les corpus de production, et leurs perceptions subjectives explicites. La question qui se pose est celle de l'écart entre usages et représentations : la variabilité des performances des locuteurs que la dessine la sociolinguistique de la liaison en Côte d'Ivoire est-elle parfaitement superposable aux perceptions individuelles que les Ivoiriens déclarent avoir au sujet du phénomène?

Le troisième objectif que nous nous proposons est de comparer les résultats de cette enquête avec l'étude perceptive effectuée à Paris (Celata, De Flaviis et Floquet, à paraître). Dans leur corpus, les auteurs constatent que l'absence d'une liaison variable produit des réactions diversifiées qui ne sont prévisibles ni à partir de la classification traditionnelle, ni à partir de données de production; aussi observent-ils un niveau de conscience métaphonologique assez bas, qu'ils interprètent comme étant strictement lié à la variabilité intrinsèque de la liaison. Les auteurs concluent enfin à l'existence d'un « un écart entre ce que les locuteurs font, dans leur production, et ce que les locuteurs pensent qu'il est acceptable de faire - ou autrement dit, entre ce qu'ils font, et ce qu'ils pensent faire » (Celata, De Flaviis et Floquet, à paraître).

Dans le paragraphe qui suit nous allons localiser de façon très succincte et les principaux facteurs sociolinguistiques et socio-psychologiques que nous estimons pouvoir peser sur les réponses des informateurs.

\subsection{Quelques fonctions et représentations du français en Côte d'Ivoire qui peuvent affecter les perceptions explicites de la liaison}

Parmi les francophonies de l'Afrique subsaharienne, la Côte d'Ivoire est regardée comme le pays le plus francophone (Lafage 1991 ; Kube 2005). Le français y est omniprésent : il remplit les fonctions de langue officielle et langue véhiculaire, il est la seule langue d'enseignement et occupe le terrain dans toute forme d'interaction sociale. Les Ivoiriens s'en approprient suivant différents modes : acquisition simultanée avec une autre langue ; apprentissage par l'école; apprentissage par les enfants non scolarisés et par les adultes comme langue seconde en milieu informel (urbain surtout). Comme le souligne Boutin (1998), contrairement à d'autres pays subsahariens francophones, le français en Côte d'Ivoire n'a jamais été en situation de diglossie ou en compétition avec une des soixantequatre langues locales qui occupent le terrain. Ce prestige unique de la langue française en Côte d'Ivoire est lié en partie à des choix politiques précis de la part des autorités: dès l'indépendance, les gouvernements locaux ont mis en œuvre une politique linguistique exoglossique (Kube 2005) qui a sollicité le français comme vecteur de promotion sociale. Aujourd'hui, la langue française constitue une importante clé sociale et «confère des 
pouvoirs exorbitants et ceux qui en profitent n'ont, bien entendu aucune envie de les perdre » (Abia Aboa 2008).

Le premier facteur que nous voyons comme un possible moteur agissant sur les perceptions individuelles de la liaison, est le fait que le français remplit dans ce pays une fonction essentiellement utilitaire : dans le scénario plurilingue et multiethnique ivoirien cette langue assure les échanges communicationnels. Cette fonction véhiculaire aurait un fort impact sur les attitudes plus ou moins tolérantes que les locuteurs peuvent avoir envers les écarts du standard. Comme le montre Boutin (2002), "si dans un premier temps, les écarts du français châtié étaient perçus, par la première génération d'enseignants comme des "fautes graves " à corriger, certains de ces écarts ont été néanmoins, peu à peu légitimés par le plus grand nombre ».

Le deuxième aspect qui nous intéresse ici est le sentiment de revendication identitaire que pourraient éprouver les Ivoiriens face à une langue coloniale qui ne répond pas à leurs besoins expressifs. À ce propos, Abia Aboa souligne qu'il faudrait «faire une distinction entre appropriation linguistique et appropriation identitaire, car l'usage quotidien d'une langue et son adaptation par des locuteurs à leurs propres besoins linguistiques ne permettent pas encore de dire si cette langue est perçue par les locuteurs comme étant leur propre langue ». L'existence du «français populaire ivoirien » (FPI) témoignerait justement d'une tentative de modeler et adapter la langue importée par les anciens colons aux formes de la société ivoirienne. À cela doit s'ajouter la portée symbolique du nouchi (Boutin et Kouadio N'Guessan 2015) : «Le nouchi remplit, pour les élèves, des fonctions communicatives et identitaires que ni le français qu'ils apprennent tous à l'école, ni les langues ivoiriennes de leur répertoire linguistique ne peuvent satisfaire et qui vont bien au-delà des fonctions attribuées à cette langue des jeunes » (Abia Aboa 2013).

Un troisième facteur à considérer dans le cadre de notre recherche peut être ramené la distinction que posent les sociolinguistes entre une "norme exogène » (le français de France) supposée être le modèle normatif véhiculé par l'école et une " norme endogène », que nous considérons avec Manessy 1992 comme «l'usage auquel les locuteurs jugent normal de recourir dans les circonstances où la pression de la norme exogène est sinon supprimée, du moins suspendue et où la vigilance métalinguistique se trouve en quelque sorte mise en veilleuse $»$.

«Ce que nous désignons par le terme peut-être impropre de « norme endogène " n'est pas un ensemble de règles ou de conventions explicitables, ce qui impliquerait la reconnaissance au moins implicite d'une normativité, mais une normalité fondée sur le désir réciproque de communiquer, un accord implicite quant à l'adéquation des modes d'expression et un savoir culturel partagé » (Manessy 1992).

De toute évidence, à tout ces facteurs il faut ajouter le poids de l'insécurité linguistique qui caractérise plus ou moins fortement tous les Francophones hors Europe.

\subsection{La distribution de la liaison en Côte d'Ivoire : un bref rappel}

D'après la définition de Gadet (1997), la liaison est un indicateur sociolinguistique fort : suivant le registre de parole, l'âge, le statut professionnel, l'origines géographique des locuteurs, la plupart des liaisons peuvent apparaître ou disparaître de façon variable et imprédictible. Le corpus PFC nous fournit un outil précieux pour décrire la sociophonétique du phénomène et pour mieux comprendre les dynamiques à la base de son comportement différencié dans l'espace francophone. Les descriptions récentes qui ressortent de l'analyse de corpus attestent aussi bien en France que dans les points d'enquête africains que la liaison catégorique (toujours réalisée) est restreinte à un petit nombre de contextes; les 
liaisons variables en revanches ont lieu dans un plus grand nombre de contextes (Durand et al. 2011 ; Bordal et Lyche 2008).

Les études quantitatives sur la liaison en Côte d'Ivoire ne sont pas très nombreuses. Nous en donnons un aperçu très simplifié. L'analyse de Boutin et Turcsan (2009) montre que le comportement de la liaison en Côte d'Ivoire présente peu de particularités par rapport aux tendances hexagonales: le phénomène est toujours réalisé après un clitique (en, on, un, des, les, vous, etc.) mais il est variable ou très rare dans la plupart des contextes (Boutin 2014). En plus, conformément aux tendances observées dans les autres pays africains francophones (Bordal et Lyche 2008) les liaisons variables sont beaucoup plus rares dans ce pays qu'en France. Le registre de parole a une incidence sur la variation du phénomène : le nombre de réalisations attestées en conversation augmente sensiblement à la lecture, comme le montrent les taux de réalisation après est où la liaison a lieu dans $50 \%$ des cas à la lecture mais seulement $5 \%$ en parole spontanée. La même variation s'observe pour les liaisons après les noms pluriels, qui ne sont jamais réalisées en contexte de parole spontanée, mais qui apparaissent (très rarement) à la lecture. Après les monosyllabiques dans, chez, très la réalisation est encore une fois variable; enfin, aucune liaison épenthétique ou interdite n'est attestée dans le corpus d'entretiens PFC. Dans les paragraphes qui suivent nous allons intégrer ces données avec les résultats de notre étude perceptive à Abidjan: la photographie sociolinguistique que nous fournit PFC est-elle superposable à celle des réactions linguistiques des locuteurs ivoiriens?

\section{Le test}

En 2019 nous avons eu l'occasion de prendre partie aux phases de codage et d'analyse d'une étude perceptive analogue effectuée à Paris. Cette expérience nous a permis de réfléchir sur les points critiques du protocole et les possibilités d'amélioration de la méthode : tout en gardant intacts les objectifs, les questions de recherche, les fondements théoriques et une bonne partie de la procédure, la méthode que nous allons présenter dans cet article veut être une version standardisée et plus fine de la précédente.

\subsection{Matériel et corpus}

Notre expérience se base sur le texte PFC «Le premier ministre ira-t-il à Beaulieu ?». Préalablement à l'expérience, nous avons demandé à un locuteur natif francophone de lire le texte en introduisant des déviations par rapport à la norme standard. Les déviations de liaison étaient les suivantes: nous avons prononcé [nuavõ]; les opposants prononcé

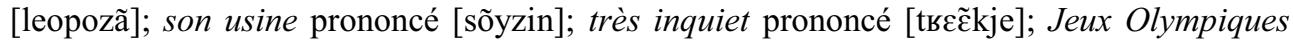
prononcé [3øolẽpik]; grand émoi prononcé [gкãdemwa]; visites officielles prononcé [vizitofisjel]; pâtes italiennes prononcé [patitaljen]; comment, en plus prononcé [komãtãplys].

L'enregistrement sonore employé pour les enquêtes précédentes contenait aussi des distracteurs qui étaient au nombre de quatre : décidé prononcé [deside]; le hazard prononcé [lazas]; paru prononcé [pari]; jeûne prononcé [zœn], auxquels nous avons ajouté fin prononcé [f̃̃jn]; étaient prononcé [ete]; villes prononcé [vij]; côte prononcé [kot] ; la haine prononcé [len]; préfecture prononcé [prefektsyR] ; déclaré prononcé [deklaRe];

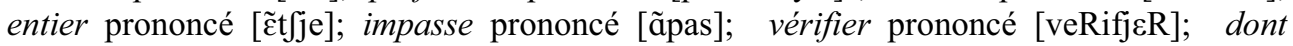
prononcé [dã].

Le test a été administré à une population de 22 étudiants universitaires natifs d'Abidjan n'ayant pas reçu une formation spécifique en linguistique. Lors de l'expérience les informateurs disposaient du texte imprimé.

\subsection{Méthode}

Le protocole se divise en deux temps. Dans un premier temps, nous avons demandé aux informateurs d'écouter l'enregistrement sonore et de marquer sur le papier les contextes 
qu'ils jugeaient notables ou déviants par rapport à leurs attentes de locuteurs natifs. Dans un deuxième temps nous leur avons demandé de commenter et justifier spontanément leurs opinions.

En continuité avec le protocole administré à Paris, nous avons calculé le pourcentage des cas où les sujets n'ont pas repéré la déviation ainsi que les pourcentages des cas où ils l'ont repérée et jugée (i) inacceptable, (ii) partiellement inacceptable (c'est-à-dire que le jugement d'inacceptabilité a été exprimé avec des hésitations ou des changements d'avis tout au long du commentaire), (iii) partiellement acceptable (jugement d'acceptabilité exprimé avec des hésitations ou des changements d'avis) ou (iv) acceptable.

Le codage des réponses métaphonologiques, reprend la grille codée de Pinto et El Euch (2015) qui permet d'attribuer une valeur numérique aux justifications explicites. Tout comme dans le travail de Celata, De Flaviis et Floquet (à paraître), nous avons attribué « 0 » aux réponses tautologiques ou défaillantes du type: «je ne sais pas », " c'est bizarre », «ce n'est pas français». Nous avons attribué «1» quand l'explication grammaticale était amorcée (mais pas complète) et faisait référence à des critères normatifs («on nous a appris comme ça »), géographiques ( «c'est une question de là où on vit»), phonologiques («pour aller plus vite, c'est plus fluide») ou lexicaux («c'est une expression figée / on dirait un autre mot »). Nous avons attribué «2» enfin aux explications plus abouties qui présentait au moins deux explications faisant référence à des catégories abstraites : par exemple, " on m'a toujours dit $_{1 / \text { normative }}$ que c'est un $<t>$ muet $_{2 / \text { phonologique }}$ ",

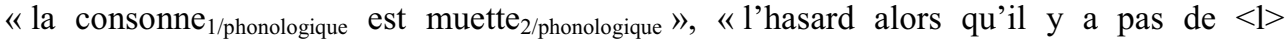
apostrophe $_{1 / \text { graphique }}$ parce qu'il y a un $<\mathrm{h}>$ muet $_{2 / \text { phonologique }} \gg$.

\subsection{Résultats}

Les résultats qui font objet de ce travail se réfèrent aux réponses épiphonologiques et métaphonologiques concernant uniquement la liaison. Pour que l'analyse soit complète, il faudrait intégrer ces premières données avec les résultats concernant les distracteurs, qui offrent une comparaison entre la perception subjective d'un phénomène multidimensionnel et complexe telle que la liaison et celle de phénomènes purement phonologiques. Ce deuxième point reste à faire.

\subsubsection{Réponses épiphonologiques pour les contextes de liaisons: analyse comparée Abidjan/Paris}

Commençons par l'analyse des réponses épilinguistiques observées dans le corpus abidjanais. Pour chaque item le Tableau 1. montre la typologie de liaison décrite dans PFC et les pourcentages des réponses "Inacceptable », "Inacceptable avec hésitation », "Acceptable avec hésitation », "Acceptable », "Non repéré ». Dans le Tableau 2, en revanche, sont comparés les pourcentages des réponses "Inacceptable » des corpus abidjanais et parisien.

\begin{tabular}{lllllll}
\hline \multicolumn{7}{c}{ Abidjan: réponses épilinguistiques (\%) } \\
\hline Item & $\begin{array}{l}\text { Typologie } \\
\text { de liaison }\end{array}$ & Inacc. & $\begin{array}{l}\text { Inacc. } \\
\text { Hésitation }\end{array}$ & $\begin{array}{l}\text { Acc.- } \\
\text { Hésitation }\end{array}$ & Acc. & $\begin{array}{l}\text { Non } \\
\text { repérés }\end{array}$ \\
\hline nous\#avons & Cat & 41 & $\mathbf{0}$ & $\mathbf{9}$ & $\mathbf{9}$ & 41 \\
les\#opposants & Cat & 18 & $\mathbf{0}$ & $\mathbf{5}$ & $\mathbf{5}$ & $\mathbf{7 3}$ \\
son\#usine & Cat & $\mathbf{1 8}$ & $\mathbf{0}$ & $\mathbf{0}$ & $\mathbf{0}$ & $\mathbf{8 2}$ \\
très\#inquiet & Var & $\mathbf{1 4}$ & $\mathbf{9}$ & $\mathbf{5}$ & $\mathbf{0}$ & $\mathbf{7 3}$ \\
Jeux\#Olympiques & Var & $\mathbf{0}$ & $\mathbf{5}$ & $\mathbf{0}$ & $\mathbf{0}$ & $\mathbf{9 5}$ \\
\hline
\end{tabular}




\begin{tabular}{lllllll}
\hline granD émoi & Var & $\mathbf{5}$ & $\mathbf{0}$ & $\mathbf{0}$ & $\mathbf{0}$ & $\mathbf{9 5}$ \\
visites\#officielles & Var & $\mathbf{0}$ & $\mathbf{0}$ & $\mathbf{0}$ & $\mathbf{5}$ & $\mathbf{9 5}$ \\
pâtes\#italiennes & Var & $\mathbf{0}$ & $\mathbf{0}$ & $\mathbf{0}$ & $\mathbf{0}$ & $\mathbf{1 0 0}$ \\
commenT en plus & Imp & $\mathbf{5 5}$ & $\mathbf{0}$ & $\mathbf{0}$ & $\mathbf{0}$ & $\mathbf{4 5}$ \\
\hline
\end{tabular}

Tableau 1. Pourcentage des réponses épilinguistiques concernant les contextes de liaison (Abidjan).

\begin{tabular}{ll|l}
\hline & \multicolumn{2}{l}{ Réponses « Inacceptable » $\mathbf{( \% )}$} \\
\hline Item & Abidjan & Paris \\
\hline nous\#avons & $\mathbf{4 1}$ & 68 \\
les\#opposants & $\mathbf{1 8}$ & 64 \\
son\#usine & $\mathbf{1 8}$ & 53 \\
très\#inquiet & $\mathbf{1 4}$ & 63 \\
Jeux\#Olympiques & $\mathbf{0}$ & 42 \\
granD émoi & $\mathbf{5}$ & 42 \\
visites\#officielles & $\mathbf{0}$ & 16 \\
pâtes\#italiennes & $\mathbf{0}$ & 0 \\
commenT en plus & $\mathbf{5 5}$ & 53 \\
\hline
\end{tabular}

Tableau 2. Pourcentage des réponses « Inacceptable » à Abidjan et à Paris.

Nous notons qu'à Abidjan les items non repérés sont très nombreux : la moyenne des pourcentages des non repérages atteigne $77 \%$. Cette tendance se heurte aux résultats de l'enquête parisienne où, au contraire, les auteurs attestent des taux de repérage et de censure bien plus élevés. Pour les contextes de liaison classés comme catégoriques la comparaison des deux corpus montre des résultats contradictoires : si on se limitait aux données de l'usage, nous avons, son usine et les opposants, on pourrait s'attendre à une censure presque totale, comme cela a été le cas pour Paris (respectivement 68\%,64\%, 53\%); à Abidjan par contre, la non acceptation est très variable et semble se différencier essentiellement suivant la catégorie grammaticale du mot liaisonnant : dans le contexte Pronom sujet+verbe (nous avons) la censure est plus nette $41 \%$ que pour le contexte Dét+nom (les opposants et son usine) où seuls $18 \%$ déclarent ne pas accepter l'absence de liaison. Nous constatons également que les taux de non repérage sur ces trois contextes sont très élevés : $41 \%$ pour nous avons, $73 \%$ pour les opposants et $82 \%$ pour son usine. Sur comment en plus, nous nous attendions à une censure totale, étant donné qu'après comment la liaison n'est jamais faite dans le corpus ivoirien PFC. Cependant, la non acceptation n'atteigne que 55\%, tout comme à Paris.

Quant aux réponses épilinguistiques concernant les cinq autres contextes classés comme variables mais peu fréquents (et moins fréquents qu'en France), nous ne constatons pas une grande fluctuation. À Paris, le manque de liaison sur très inquiet est majoritairement perçu comme déviant (63\%). À Abidjan par contre, seuls $14 \%$ des locuteurs déclarent ne pas accepter cette prononciation malgré cette liaison soit fréquente (76\% selon Boutin 2012). En revanche, les déviations sur les contextes Nom pluriels+ adj (Jeux Olympiques, visites officielles, pates italiennes) où les réalisations attestées dans PFC sont très basses (11\%) sont presque complètement inaperçues et aucun des informateurs ne les qualifie d'inacceptables. Le Tableau 2 montre qu'à Paris les réponses concernant ce groupe d'item item sont très différentes, du moins pour Jeux olympiques et visites officielles où la censure 
atteigne respectivement $42 \%$ et $16 \%$. Dans le cas des liaisons variables, la perception à Abidjan semble être plus conforme aux pratiques attestées dans les corpus de parole. Pour les liaisons catégoriques en revanche, nous constatons un écart majeur entre les données de l'usage et l'acceptation des formes déviantes.

\subsubsection{Réponses métaphonologiques pour les contextes de liaison: analyse comparée Abidjan/Paris}

Les résultats des réponses métaphonologiques divisés par sujet sont montrés dans la Figure 1. Nous avons analysé les commentaires concernant les 9 contextes de liaison, donc la valeur maximale était 18 (9 items multipliés par le score 2 ).

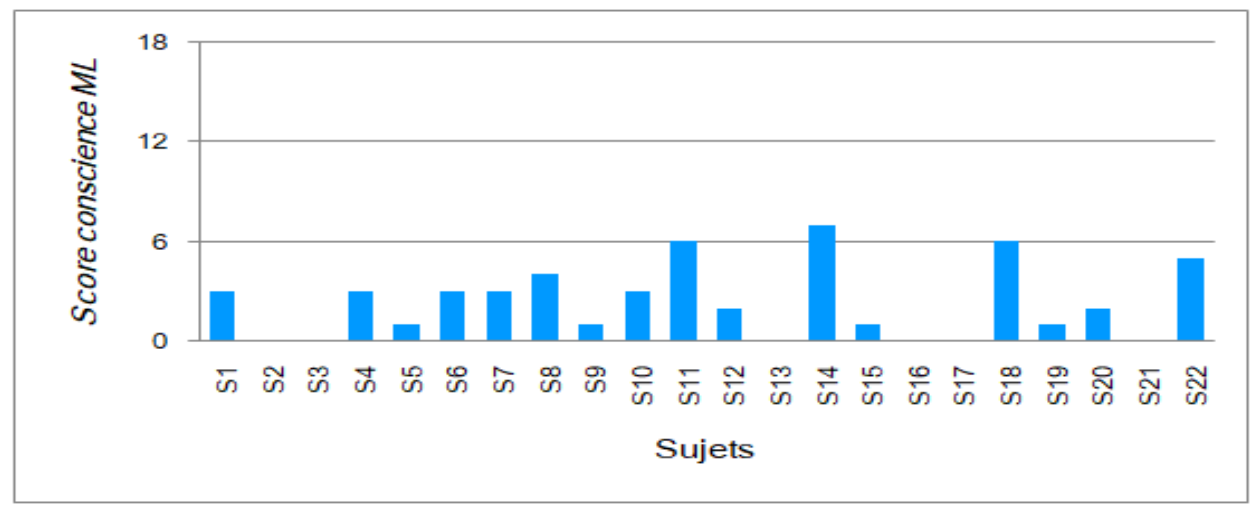

Figure 1. Scores métaphonologiques réalisés par chaque locuteur sur la totalité des contextes de liaison (Abidjan).

La moyenne des scores égale 2,3 : elle est donc très basse et se distingue des scores calculés sur la populations parisienne qui égale 5. Cependant, il faut souligner que sur cette moyenne pèse fortement sur le taux élevé des items non repérés qui, comme nous l'avons vu, correspond à environ $80 \%$. Quand les informateurs ont repéré la déviation, seule une minorité d'entre eux a donné une réponse tautologique ou inexistante (dont la valeur serait donc $« 0 »)$.

\section{Discussion et conclusions}

Les résultats des réponses épilinguistiques montrent que l'acceptabilité de la liaison ne correspond que partiellement aux données de production attestées dans PFC : si l'absence des liaisons variables ou rares n'est pas perçue comme déviante par la majorité des locuteurs, les liaisons toujours réalisées donnent lieu, en revanche, à une acceptation variable qui semble se différencier suivant la catégorie grammaticale du mot liaisonnant. Comparés aux données de Paris, les résultats montrent que les étudiants universitaires abidjanais légitiment davantage les réalisations « déviantes » des liaisons et manifestent une attitude plus libre et tolérante. Les exemples de les opposants, très inquiet et Jeux oplympiques le montrent. Les raisons à cela pourraient être interprétées à nos yeux comme la conséquence directe d'une représentation fonctionnelle et pragmatique de la langue (comme le montre de travail de Lachet 2014 centré sur les représentations grammaticales des étudiants universitaires) et d'une réalité sociolinguistique particulière enracinée dans une dimension orale et plurilingue. Elles semblent également être liées aux exigences de communication interethnique et à un scénario socioculturel où cohabitent différents modes d'appropriation du français et la reconnaissance d'une «norme endogène » de la part de la communauté linguistique. 
Les scores de conscience méta- sont également assez bas. De l'observation des commentaires émerge que les argumentations des informateurs se basent soit sur des connaissances explicites de la règle phonologique (avec une référence constante à la norme véhiculée par l'école) soit, encore une fois, sur les exigences de se comprendre mutuellement. Les justifications explicites que nous avons observées oscillent entre deux attitudes opposées: l'une, plus tolérante, qui privilégie la réussite de l'échange verbal et légitime les écarts du modèle normatif, l'autre (minoritaire), plus orthodoxe et normative, qui est capable à donner une justification plus ou moins exhaustive des règles apprises à l'école et qui manifeste une légère stigmatisation des pratiques non conformes. Par exemple, à propos des déviations sur très inquiet et son usine un des informateurs déclare : «c'est pas faux mais les gens diront qu'il a mal parlé parce qu'il a pas fait la liaison »; «c'est pas faux mais les autres à coté de lui ils vont se moquer ». Pour une partie des sujets donc prononcer les liaisons variables semble être une bonne pratique susceptible de protéger les locuteurs d'une marginalisation sociale ou d'une dépréciation de leur façon de parler.

Finalement, les savoirs ordinaires occupent désormais une place centrale dans l'étude des faits linguistiques (Paveau 2008 ; Vicari 2016). Notre enquête en Côte d'Ivoire nous a permis d'analyser les appréciations spontanées des locuteurs non experts au sujet de la liaison et de quantifier leurs justifications explicites. L'approche herméneutique de la liaison et la démarche hybride (qualitative et quantitative) que nous avons adoptées dans cette recherche nous permettent d'étudier la variation du phénomène suivant une échelle d'observation nouvelle qui s'interroge non seulement sur les pratiques linguistiques externes mais aussi sur la diversité des perceptions individuelles sous-jacentes. Une analyse de la liaison dans l'espace francophone par corpus d'intuitions naïves pourrait constituer, à notre avis, un instrument informatif très utile pour observer la diversité des connaissances linguistiques des locuteurs sur lesquelles reposent les motivations sous-jacentes à leurs pratiques. 


\section{Références bibliographiques}

Abia Aboa, A. L. (2008). La Francophonie ivoirienne. Documents pour l'histoire du français langue étrangère ou seconde [En ligne].

Abia Aboa, A.-L. (2013). Langue française et identité culturelle ivoirienne : [en ligne].

Bordal, G. et Lyche, C. (2008). La liaison en terre africaine, Journées PFC.

Boutin, B.A. et Turcsan, G. (2009). La prononciation du français en Afrique : la Côte d'Ivoire, Durand et al. (éds), Phonologie, variation accents du français, Hermès.

Boutin, B.A. ( 2002). Description de la variation : études transformationnelles des phrases du français de Côte d'Ivoire. Thèse de Doctorat, Université de Grenoble III.

Boutin, B. A. (1998). Quelques variantes syntaxiques du français en Côte d'Ivoire. Mémoire de DEA, Université de Stendhal, Grenoble III.

Boutin, B. A. et Kouadio N'Guessan, J. (2015). Le nouchi c'est notre créole en quelque sorte, qui est parlé par presque toute la Côte d'Ivoire : [en ligne].

Boutin, B.A. (2014). Liaisons en français et terrains africains. Durand, J., Kristoffersen, G. et Laks, B., avec la collaboration de J. Peuvergne (dir.), La phonologie du français: normes, périphéries, modélisation. Nanterre: Presses Universitaires de Paris Ouest, pp. 153-172

Celata C., De Flaviis G. et Floquet O. (à paraitre). Pour une approche herméneutique de la liaison: les discours épilinguistiques et métalinguistiques des étudiants universitaires parisiens. Journal of Applied Psycholinguistics.

Chard D., et Dickson S. (1999). Phonological Awareness: Instructional and Assessment Guidelines. Intervention in School and Clinic; 34, 5, pp. 261-270.

Durand, J., Laks, B. \& Lyche, C. (2002). La phonologie du français contemporain: usages, variétés et structure. in Pusch C. et Raible W. (éds.). RomanistischeKorpuslinguistikKorporaundgesprocheneSprache/Romance Corpus Linguistics - Corpora and Spoken Language. Tübingen: GunterNarrVerlag, 93-106.

Durand, J., Laks, B., Calderone, B., \& Tchobanov, A. (2011). Que savons-nous de la liaison aujourd'hui?. Langue française 169, 103-126.

Eychenne, J., \& Laks, B. (2017), La liaison en français contemporain: normes, usages, acquisitions. Journal of French Language Studies, 27, 1-12.

Floquet, O. \& Laks, B (2017). Liaison et acceptabilité: une étude pilote. Repères-Dorif, 12, [en ligne].

Gadet, F. (1997) : Le français ordinaire. Paris : Armand Colin.

Gombert, J. É. (1990). Le développement métalinguistique. Paris : PUF.

Kube, S. (2005). La francophonie vécue en Côte d'Ivoire. Paris: L'harmattan.

La Mantia, F. (2017). "Un atteggiamento irenico". Su alcune pagine culioliane di Tullio De Mauro. Bollettino del centro di Studi Filologici e Linguistici Siciliani, 28, 151-174.

Lachet, C., (2014). Pratiques et représentations grammaticales des étudiants à l'université. Actes du 2e Colloque international du GRAC sous la direction de Jean-Michel Kalmbach et Sofia Stratilaki-Klein, pp. 70-81.

Lafage, S. (1991). L'argot des jeunes Ivoiriens, marque d'appropriation du français ? Langue française, $90: 95-105$.

Laks, B., Calderone, B. \&Celata, C. (2018). French Liaison in the Light of Corpus Phonology: From Lexical Information to Patterns of Usage Variation, in Brentari D. et Lee J. (éds.). Shaping Phonology, Chicago: The University of Chicago Press, 278-308.

Laks, B., Calderone, B. et Celata, C. (2014). French Liaison and the lexical repository. Celata S. et Calamai S. (éds.). Advances in Sociophonetics, Amsterdam: John Benjamins Publishing Company, 31-56.

Manessy, G. (éd.) (1992). Norme endogène et normes pédagogiques en Afrique noire francophone ", in Multilinguisme et développement dans l'espace francophone : 43-81, Coll. Langues et développement, Paris : Didier Érudition.

Paveau, M.-A. (2008). Le parler des classes dominantes, objet linguistiquement incorrect? Dialectologie perceptive et linguistique populaire. Ela. Études de linguistique appliquée, 150, pp. $137-156$

Pinto, M. A., \& El Euch, S. (2015). La conscience métalinguistique. Québec : P.U.L. 
Soum-Favaro, C., Coquillon, A. \& Chevrot, J.P. (2014). La liaison: approches contemporaines. Bern : Peter Lang.

Vicari, Stefano (2016). Pour une approche de la linguistique populaire en France. Roma : Aracne. 